\title{
Comment on "Comparisons with analytical solutions from Khvorostyanov and Curry (2007) on the critical droplet radii and supersaturations of CCN with insoluble fractions" by Kokkola et al. (2008)
}

\author{
V. I. Khvorostyanov ${ }^{1}$ and J. A. Curry $^{2}$ \\ ${ }^{1}$ Central Aerological Observatory, Dolgoprudny, Moscow Region, Russia \\ ${ }^{2}$ School of Earth and Atmospheric Sciences, Georgia Institute of Technology, Atlanta, Georgia, USA
}

Received: 1 April 2009 - Published in Atmos. Chem. Phys. Discuss.: 15 April 2009

Revised: 6 July 2009 - Accepted: 1 August 2009 - Published: 20 August 2009

\begin{abstract}
Analytical solutions for the critical radii and supersaturations of the cloud condensation nuclei $(\mathrm{CCN})$ with insoluble fractions were derived by Khvorostyanov and Curry (2007, hereafter KC07). These solutions generalize Köhler's solutions for an arbitrary soluble fraction of $\mathrm{CCN}$, and have two limiting cases: large soluble fraction (Köhler's original solution); and a new "low soluble fraction" limit. Similar solutions were found subsequently by Kokkola et al. (2008, hereafter Kok08); however, Kok08 used the approximation of an ideal and dilute solution, while $\mathrm{KC} 07$ used more accurate assumptions that account for nonideality of solutions. Kok08 found a large discrepancy with $\mathrm{KC} 07$ in the critical supersaturations. It is shown that the major discrepancy with $\mathrm{KC} 07$ found in Kok08 was caused by the simple mistake in Kok08, where comparison was made not with the general solution from $\mathrm{KC} 07$, but with the Köhler's solution or with some unknown quantity, not even with the "low soluble fraction" limit. If general solutions from the two works are compared, the equations from Kok08 mostly repeat the equations from $\mathrm{KC} 07$, except that Kok08 use the ideal dilute solution approximation. If the mistake in Kok08 is corrected, then the differences in the critical radii and supersaturations do not exceed $16-18 \%$, which characterizes the errors of the ideal dilute solution approximation. If the Kok08 scheme is modified following $\mathrm{KC} 07$ to account for the non-ideality of solution, then the difference with $\mathrm{KC} 07$ does not exceed 0.4$1 \%$.
\end{abstract}

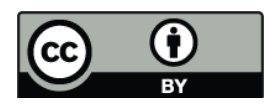

Correspondence to: J. A. Curry (curryja@eas.gatech.edu)

\section{Introduction}

A theoretical basis for consideration of hygroscopic growth of atmospheric aerosols or cloud condensation nuclei (CCN) and their activation into cloud drops was provided by the Köhler (1936) equation that enabled prediction of the CCN critical radii $r_{c r}$ and supersaturations $s_{c r}$ for drop activation. Kohler's equation was designed originally for fully soluble aerosols. However, natural aerosols are almost always mixed, i.e., contain soluble and insoluble fractions, and Köhler's equation was modified later to include insoluble fractions (e.g., Hänel, 1976; Pruppacher and Klett, 1997, hereafter PK97; Charlson et al., 2001; Ghan et al., 1993, 1995; Kulmala et al., 1993; Levin et al., 1996; Shulman et al., 1996; Laaksonen et al., 1998; Seinfeld and Pandis, 1998; Lohmann, and Feichter, 2005; McFiggans et al., 2006; Khvorostyanov and Curry, 2006, 2008; Foster et al., 2007).

Simple analytical solutions for $r_{c r}$ and $s_{c r}$ are desirable for understanding the parametric dependencies and also for developing cloud activation parameterizations for cloud and climate models. Such analytical solutions were derived by Khvorostyanov and Curry (2007, hereafter KC07) with sufficiently general assumptions and for arbitrary mass soluble fractions. It was shown in $\mathrm{KC} 07$ that the new equations for $r_{c r}$ and $s_{c r}$ transform into the classical Köhler's equations for sufficiently high soluble fraction, and yield a new analytical "low soluble fraction limit" for very small masses of soluble fractions where the classical equations fail. The accuracy of the new equations was verified in $\mathrm{KC} 07$ by comparison with experimental data, and previous particular cases.

Kokkola et al. (2008, hereafter Kok08) subsequently published a paper where similar analytical solutions were found

Published by Copernicus Publications on behalf of the European Geosciences Union. 
for $r_{c r}$ and $s_{c r}$ for mixed CCN. However, Kok08 used the approximations of an ideal and dilute solution, which often is not justified for CCN with insoluble fractions (e.g., PK97; Lohmann, and Feichter, 2005; McFiggans et al., 2006; Foster et al., 2007). A comparison of the critical supersaturations for a $\mathrm{NaCl}$ particle was performed in Kok08 that revealed a discrepancy with $\mathrm{KC} 07$ of up to $100 \%$, especially for large soluble fraction. Kok08 explained this discrepancy by an "assumption" made in $\mathrm{KC07}$, but it was not clear what assumptions could cause such a great discrepancy. The comparisons with Kok08 are made and possible reasons for this difference are analyzed here, including: 1) approximations in basic equations; 2) the cubic equations for the critical radii; 3) analytical solutions of these equations; 4) numerical solutions. Finally, it is determined that the major discrepancy between Kok08 and KC07 was caused by an elementary mistake in Kok08, whereby the Köhler's or some unknown quantity (not even the "low soluble fraction limit") were used in Kok08 for comparison instead of the general solution from $\mathrm{KC} 07$. The accuracy of an ideal dilute solution approximation used in Kok08 is estimated, and it is found that the use of a non-ideal solution approximation as in $\mathrm{KC} 07$ improves the accuracy.

\section{Comparison of $\mathrm{KC07}$ and Kok08}

\subsection{Comparison of the basic equations in both ap- proaches}

$\mathrm{KC} 07$ begin their derivation with the equations given in PK97 for water saturation ratio $S_{w}$ or for supersaturation $s_{w}=S_{w}-1$

$s_{w}=\exp \left(\frac{A_{k}}{r}-\frac{B}{r^{3}-r_{d}^{3}}\right)-1$,

$A_{k}=\frac{2 M_{w} \sigma_{s a}}{R T \rho_{w}}, \quad B=\frac{3 v_{s} \Phi_{s} \varepsilon_{m} m_{d} M_{w}}{4 \pi M_{s} \rho_{w}}$.

Here $A_{k}$ is the Kelvin curvature parameter, $M_{w}$ is the molecular weight of water, $\sigma_{s a}$ is the surface tension at the drop solution-air interface, $R$ is the universal gas constant, $T$ is the temperature (in degrees Kelvin), $\rho_{w}$ is the water density, $v_{s}$ is the number of ions in solution, $\Phi_{s}$ is the osmotic potential, $\varepsilon_{m}=m_{s} / m_{d}$ is the mass soluble fraction, $r$ is the drop radius, $r_{d}$ and $m_{d}$ are the radius and mass of a dry aerosol particle (CCN), $m_{s}$ and $M_{s}$ are the mass and molecular weight of the soluble fraction. The parameter $B$ describes effects of the soluble fraction and $\mathrm{KC} 07$ parameterized it as

$B=b r_{d}^{2(1+\beta)}$

where the parameters $b$ and $\beta$ depend on the chemical composition and physical properties of the soluble part of an aerosol particle. For $\beta=0.5$ and $\beta=0$, the soluble fraction is proportional to the volume $\left(B \sim \mathrm{m}_{s} \sim r_{d}^{3}\right)$ and surface area $\left(B \sim \mathrm{m}_{s} \sim r_{d}^{2}\right.$ ) respectively. Note that $\mathrm{KC} 07$ do not assume that $m_{s} \sim r_{d}^{3}$ in the $B$ term as in most other works; this allows consideration not only of $\mathrm{CCN}$ with soluble fraction mixed in the volume, but also $\mathrm{CCN}$ with surface soluble shells covering insoluble cores (e.g., Levin et al., 2006). The KC07 equations do not imply a dilute or ideal solution approximation.

Kok08 begin their derivation using also Köhler's equation with insoluble fraction, but using another approximation

$s_{w}=\exp \left(\frac{A_{F}}{D_{p}}-\frac{B_{F}}{D_{p}^{3}-D_{p, 0}^{3}}\right)-1$,

where $D_{p}$ is the drop diameter, $D_{p, 0}$ is the equivalent diameter of the insoluble core (instead of the dry particle). The parameters $A_{F}$ and $B_{F}$ used by Kok08 are taken from Seinfeld and Pandis (1998, hereafter SP98), where they are specified as

$A_{F}=\frac{4 M_{w} \sigma_{w}}{R T \rho_{w}}, \quad B_{F}=\frac{6 n_{s} M_{w}}{\pi \rho_{w}}$

where $n_{s}$ is the number of solute moles in a drop. It is easily shown that when the dilute solution approximation is applied to $B$, then

$A_{F}=2 A_{K}, \quad B_{F}=8 B$.

Note, however, that the form of the denominator in the 2nd term in Eq. (4) $\left(D_{p}^{3}-D_{p 0}^{3}\right)$ and of $B_{F}$ in Eq. (5) used in Kok08 are given in SP98 as an approximation for ideal dilute solutions only. In the dilute approximation, the activity coefficient $\gamma_{w}$ or osmotic coefficient $\Phi_{s}$ in Eq. (2) tend to unity (therefore they are absent in Eq. 5), the volume occupied by solute can be neglected relative to the droplet volume, and the volume of the wet drop is much greater than the original dry volume. These limitations may become invalid for $\mathrm{CCN}$ with small soluble fractions and solutions are not dilute particularly at the time of drop activation, and $\gamma_{w}$ or $\Phi_{s}$ can differ from 1 even at $S_{w} \rightarrow 1$ (e.g., Hänel, 1976; PK97, Table 6.3). In particular, this is illustrated by Fig. 3 in KC07, where the ratio $r_{c r} / r_{d}$ is close to 1 for small $r_{d}$ and $\varepsilon_{m}$, i.e., the solution is concentrated and nonideal. More complete expressions without assuming ideality and high dilution are given in SP98 (without coefficients $A_{F}$ and $B_{F}$ ) and in PK97 and are accounted for in $\mathrm{KC} 07$ in the coefficients $b$ in (3).

KC07's Eqs. (1) and (2) are equivalent to the nonideal approximations from SP98 and PK97 and do not assume a dilute solution. The dilute approximation used by Kok08 can be inconsistent for $\mathrm{CCN}$ with high insoluble fraction, and may lead to substantial errors because the solution may be concentrated even at high humidities. This difference in approximations is one of the reasons for the discrepancies between KC07 and Kok08. Kok08 state that KC07 "use approximation in the derivation of the critical radii". However, a comparison above of the basic equations from $\mathrm{KC} 07$ with Kok08 shows that the approximation used in $\mathrm{KC} 07$ is more accurate than the approximation used in Kok08. 


\subsection{Comparison of the cubic equations for the critical radii or diameters}

The critical radius $r_{c r}$ of a drop activation is obtained from the condition of maximum, $d s\left(r_{c r}\right) / d r_{c r}=0$, with $s_{c r}\left(r_{c r}\right)$ defined by (1). This yields a sixth-order equation in $r_{c r}$ that was reduced in $\mathrm{KC} 07$ to a cubic algebraic equation [Eq. (26) in $\mathrm{KC} 07]$

$r_{c r}^{3}+a r_{c r}^{2}-r_{d}^{3}=0$,

$a=-\left(\frac{3 B}{A_{K}}\right)^{1 / 2}=-\left(\frac{3 b r_{d}^{2(1+\beta)}}{A_{K}}\right)^{1 / 2}$.

Kok08 arrived at the similar cubic equation (Eq. 4 in Kok08) identical in form to Eq. (7); the difference is in the particle size term $D_{p, 0}$ instead of $r_{d}$ in KC07 The term $r_{d}$ in Eq. (7) is the dry radius of a CCN that includes soluble and insoluble fractions, while $D_{p 0}$ is the equivalent diameter of the insoluble fraction, which arises from using a dilute solution approximation. Thus, the difference in the results between $\mathrm{KC} 07$ and Kok08 is not caused by the form of the cubic equation for the critical radii, but could be partially due to the different meaning of the particle size terms.

\subsection{Verification of the solution for the critical radii in KC07}

The solution to Eq. (7) was found in $\mathrm{KC} 07$ in the form:

$r_{c r}=r_{d} \chi(V), \quad \chi(V)=\left[V+P_{+}(V)+P_{-}(V)\right]$,

$P_{ \pm}(V)=\left(V^{3} \pm\left(V^{3}+\frac{1}{4}\right)^{1 / 2}+\frac{1}{2}\right)^{1 / 3}$

$V=\left(\frac{b r_{d}^{2 \beta}}{3 A_{k}}\right)^{1 / 2}=\frac{1}{3 r_{d}}\left(\frac{3 B}{A_{k}}\right)^{1 / 2}$.

The critical supersaturation $s_{c r}$ can be calculated by substituting $r_{c r}$ from Eqs. (8) and (9) into (1):

$s_{c r}=\exp \left(\frac{A_{k}}{r_{c r}}-\frac{B}{r_{c r}^{3}-r_{d}^{3}}\right)-1$.

One possible reason for the discrepancy between Kok08 and $\mathrm{KC} 07$ could be if this solution, Eq. (8) for $r_{c r}$, does not satisfy Eq. (7). The validity of this solution can be proven if substitution of $r_{c r}$ from Eq. (8) into (7) satisfies this equation. Denoting the left hand side of Eq. (7) as $Z r_{d}^{3}$, and substituting Eqs. (8) and (9) into (7), we obtain:

$$
\begin{aligned}
& Z r_{d}^{3}=\left[r _ { d } ^ { 3 } \left(V^{3}+P_{+}^{3}+P_{-}^{3}+3 V P_{+}^{2}+3 V P_{-}^{2}+3 V^{2} P_{+}\right.\right. \\
& \left.\left.+3 V^{2} P_{-}+6 V P_{+} P_{-}+3 P_{+} P_{-}^{2}+3 P_{+}^{2} P_{-}\right)\right] \\
& +\left[-3 r_{d}^{3}\left(V^{3}+V P_{+}^{2}+V P_{-}^{2}+2 V^{2} P_{+}+2 V^{2} P_{-}+\right.\right. \\
& \left.\left.2 V P_{+} P_{-}\right)\right]+\left[r_{d}^{3}\right] .
\end{aligned}
$$

Each square bracket denotes each of the 3 terms in Eq. (7). The solution (8) is correct if we can prove that $Z=0$. Then the left hand side is zero, and thus equal to the RHS of (7). Dividing each term in (11) by $r_{d}^{3}$, canceling equal terms with opposite signs and regrouping, then (11) is simplified as

$Z=$

$-2 V^{3}+\left(P_{+}^{3}+P_{-}^{3}\right)+3\left(P_{+}+P_{-}\right)\left(P_{+} P_{-}-V^{2}\right)-1$.

Further simplifications can be done using the properties of functions $P_{+}$and $P_{-}$:

$P_{+}^{3}+P_{-}^{3}=2 V^{3}+1$,

$P_{+} P_{-}=V^{2}=\left(\frac{1}{9 r_{d}^{2}}\right)\left(\frac{3 B}{A_{k}}\right)$.

Substituting these relations into Eq. (12), we obtain

$Z=-2 V^{3}+\left(2 V^{3}+1\right)+3\left(P_{+}+P_{-}\right)\left(V^{2}-V^{2}\right)-1 \equiv 0$. (15)

The left hand side of Eq. (15) is equal to zero, and hence Eq. (7) is satisfied by the solution (8). No approximations were made in this proof; therefore, the $\mathrm{KC} 07$ solution is exact and without additional approximation as suggested by Kok08. Thus, the solution in $\mathrm{KC} 07$ is correct and is not a reason for the discrepancy with Kok08.

\subsection{Limiting cases with large and small insoluble frac- tions}

Based on their numerical results, Kok08 concluded that the solution in $\mathrm{KC} 07$ is correct only for very small soluble fraction, $\varepsilon_{m} \ll 1$. However, this statement is incorrect. It is seen from the definition of $V$ in Eq. (9) that for any $\varepsilon_{m} \geq 0.1$, the parameter $V \gg 1$. It was shown in $\mathrm{KC} 07$ that the solution (8) then can be expanded by $1 / V$ and has a limit for this high $V$ as (Eq. 29 in $\mathrm{KC} 07)$ :

$$
\begin{aligned}
& r_{c r} \approx \\
& r_{d}\left[V+V\left(1+\frac{V^{3 / 2}}{3}\right)+V\left(1-\frac{V^{3 / 2}}{3}\right)\right]=\left(\frac{3 B}{A_{k}}\right)^{1 / 2}, \\
& s_{c r} \approx \frac{2}{3 r_{c r}}=\left(\frac{4 A_{k}^{3}}{27 B}\right)^{1 / 2} .
\end{aligned}
$$



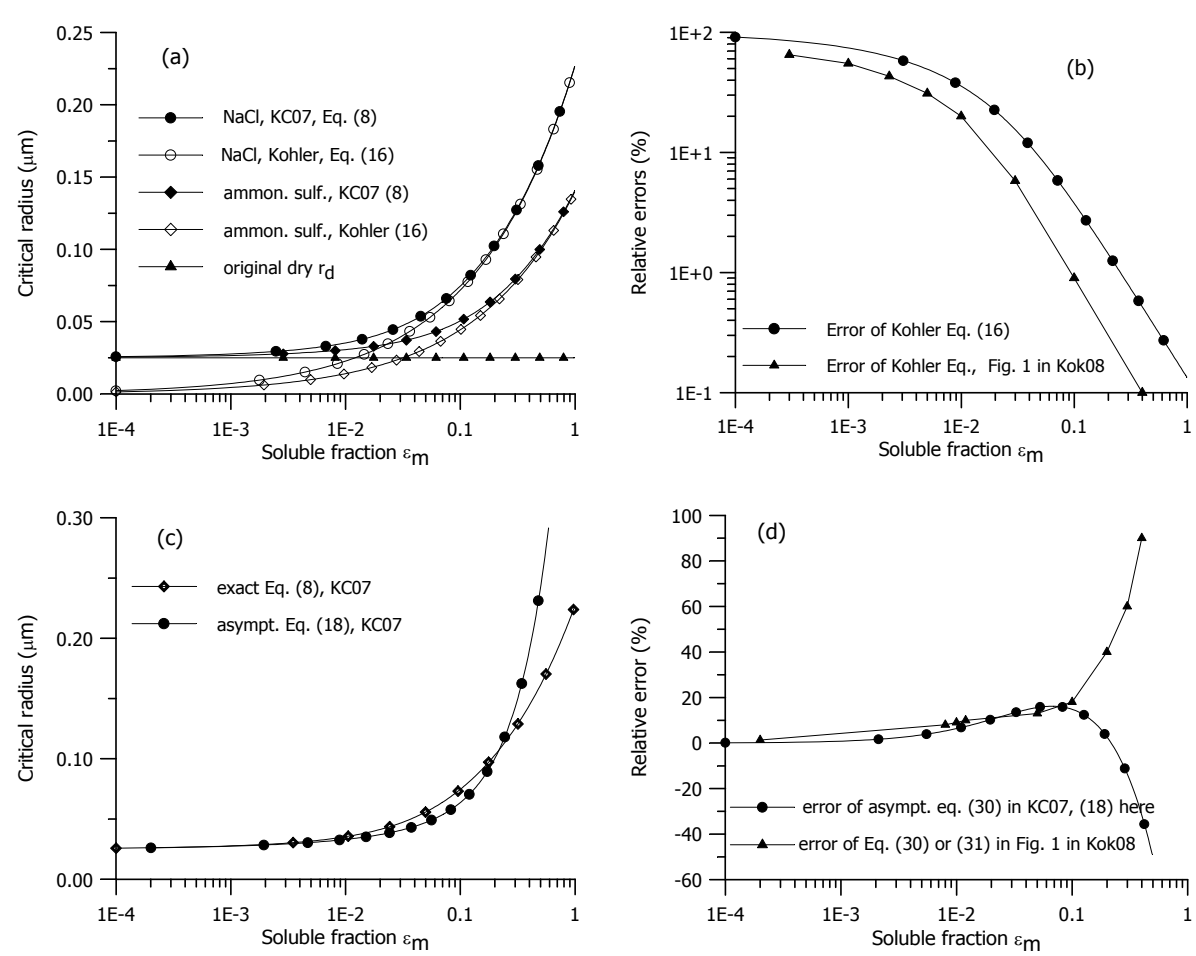

Fig. 1. Critical radii in $\mu \mathrm{m}$ (a, c) calculated with exact Eq. (8) here [(27) in KC07], and approximations (16, Kohler) and (18, "low-soluble fraction") and the relative errors in \% (b, d) of Kohler Eq. (16) here [Eq. (29) in KC07] at V》1, and asymptotic Eq. (18) [Eq. (30) in KC07] at $\mathrm{V} \ll 1$. Relative errors in (b, d) were calculated using Eqs. (8), (16), and (18) here and compared to the corresponding "dashed" and "solid" curves in Fig. 1 in Kok08.

Here, (17) follows from (16) and (10), that are the classical Köhler's expressions. Thus, the solution from KC07 gives the correct Köhler's limit for sufficiently high soluble fraction. In the opposite limit at very small soluble fractions or radii, when $V \ll 1$, another expansion of (8) by $V$ was applied and a new "low solubility" limit was obtained in KC07:

$r_{c r} \approx$

$r_{d}\left(1+V+\frac{2}{3} V^{3}\right)=r_{d}\left[1+\left(\frac{b r_{d}^{2 \beta}}{3 A_{k}}\right)^{1 / 2}+\frac{2}{3}\left(\frac{b r_{d}^{2 \beta}}{3 A_{k}}\right)^{3 / 2}\right]$.

The critical supersaturation is then calculated from the equation

$s_{c r}=\exp \left(\frac{A}{r_{c r}}-\frac{b r_{d}^{2(1+\beta)}}{r_{c r}^{3}-r_{d}^{3}}\right)-1=\exp \left(\frac{2 A}{3 r_{c r}}\right)-1$.

\subsection{Comparison of analytical solutions in $\mathrm{KC07}$ and Kok08}

Kok08 presented analytical solution for $D_{c r}$ as (notations are slightly changed here for consistency)

$D_{c r}=\frac{\alpha_{2}}{6}+\frac{2}{3}\left(\frac{3 B_{F}}{A_{F}}\right) \frac{1}{\alpha_{2}}+\frac{1}{3}\left(\frac{3 B_{F}}{A_{F}}\right)^{1 / 2}$, with $\alpha_{1}$ and $\alpha_{2}$ defined in Kok08 as

$$
\begin{aligned}
& \alpha_{2}=\left[108 D_{p 0}^{3}+8\left(\frac{3 B_{F}}{A_{F}}\right)^{3 / 2}+\alpha_{1}\right]^{1 / 3}, \\
& \alpha_{1}=12\left[81 D_{p 0}^{6}+12 D_{p 0}^{3}\left(\frac{3 B_{F}}{A_{F}}\right)^{3 / 2}\right]^{1 / 2}
\end{aligned}
$$

Simplifying the coefficients $\alpha_{1}$ and $\alpha_{2}$ and using Eq. (13) plus definitions of $P_{ \pm}$and $V$ from Eq. (9), the following relations are determined:

$\frac{\alpha_{2}}{6}=2\left[r_{d} P_{+}\left(r_{p 0}\right)\right]$,

$\frac{2}{3}\left(\frac{3 B_{F}}{A_{F}}\right) \frac{1}{\alpha_{2}}=2\left[r_{d} P_{-}\left(r_{p 0}\right)\right]$,

$\frac{1}{3}\left(\frac{3 B_{F}}{A_{F}}\right)^{1 / 2}=2\left[r_{d} V\left(r_{p 0}\right)\right]$,

where $V\left(r_{p 0}\right), P_{+}\left(r_{p 0}\right)$, and $P_{-}\left(r_{p 0}\right)$ are defined by (9), but with $r_{p 0}=D_{p 0} / 2$ instead of $r_{d}$. These equations express the 3 terms on the right hand side of Kok08's Eq. (20) through the quantities used in KC07 and defined in (9) here. Substituting them into (20), we obtain 
$D_{c r}=$

$2 r_{d}\left[V\left(r_{p 0}\right)+P_{+}\left(r_{p 0}\right)+P_{-}\left(r_{p 0}\right)\right]=2 r_{c r}\left(r_{p 0}\right)$.

The relation in (23) is based on Eq. (8) here from KC07. Thus, solutions obtained in Kok08 are identical to solutions from KC07 but with replacement $r_{d} \rightarrow r_{p 0}$ and are written in a slightly different form. It is not surprising, since these are both solutions to the incomplete cubic Cardano's equation (7). Thus, the form of the solutions cannot be the reason for discrepancy.

\subsection{Comparison of numerical calculations}

We performed calculations for the same case as in Kok08: for a particle with radius $r_{d}=0.025 \mu \mathrm{m}\left(D_{p}=50 \mathrm{~nm}\right)$, consisting of $\mathrm{NaCl}$ or ammonium sulfate (Kok08 considered only $\mathrm{NaCl}$ ) with variable soluble fraction $\varepsilon_{m}$ that was varied from $10^{-4}$ (highly insoluble $\mathrm{CCN}$ ) to 1 (fully soluble $\mathrm{CCN}$ ). Figure 1 shows critical radii calculated using the general Eqs. (8) and (9) here (Eqs. 27, 28 in KC07), compared to the limit $r_{c r \text {,high }}$ for high soluble fraction $\varepsilon_{m}$, which is the classical Köhler's

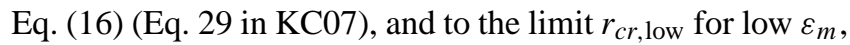
in (18) (Eq. 30 in $\mathrm{KC} 07$ ).

Figure 1a shows that the Kohler's equation for $r_{c r}$ has good accuracy at $\varepsilon_{m}>0.1-0.2$. At smaller $\varepsilon_{m}$, the accuracy of the classical equations decreases, and at $\varepsilon_{m}<0.02-0.04$, the classical equation leads to an unphysical result whereby $r_{c r}$ becomes smaller than the original dry radius $r_{d}=0.025 \mu \mathrm{m}$ (horizontal line in Fig. 1a). The curves calculated with the new KC07 solutions exceed $r_{d}$ and asymptotically approach it as $\varepsilon_{m} \rightarrow 0$. Figure 1a shows that $r_{c r}$ is quite comparable with $r_{d}$ at $\varepsilon_{m} \leq 0.01-0.03$, i.e., the mass of the critical wet $\mathrm{CCN}$ is comparable to the mass of the dry $\mathrm{CCN}$, and solution is not dilute and ideal at the time of activation, in contrast to the assumption in Kok08.

The curve $r_{c r \text {, low }}$ for "low-soluble fraction limit", Eq. (18) here, is shown in Fig. 1c. It is close to $r_{c r}$ up to $\varepsilon_{m}<0.1$, and the limit $r_{c r}$,low substantially exceeds the exact value $r_{c r}$ at larger $\varepsilon_{m}$. The error of the Kohler's equations was analyzed in detail in $\mathrm{KC} 07$. It is also illustrated in Fig. 1b here and shows that the error of Köhler's equation exceeds $5 \%$ at $\varepsilon_{m}<0.1$ and exceeds $50 \%$ at $\varepsilon_{m}<0.01$. Figure $1 \mathrm{~d}$ shows that the error of the "low- $\varepsilon_{m}$ limit" (18) grows from zero at very small $\varepsilon_{m}$ to about $20 \%$ at $\varepsilon_{m} \sim 0.1$, and exceeds $50 \%$ at $\varepsilon_{m}>0.3$.

Kok08 illustrated the "errors" of KC07 equations for supersaturation with the "dashed" and "solid" lines in their Fig. 1. In the Interactive Discussion (Kokkola, 2009, Kok09) of the ACPD version of this paper, Dr. Kokkola admitted that the calculations in Kok08 were erroneous, and this led in Kok08 to the wrong conclusions on the validity of $\mathrm{KC} 07$ equations. However, no new quantitative explanations or comparisons were given in Kok09 and the meaning of the "dashed" and "solid" lines in Fig. 1 in Kok08 remained unclear. Kok08's "dashed line" is reproduced in Fig. 1b (trian-

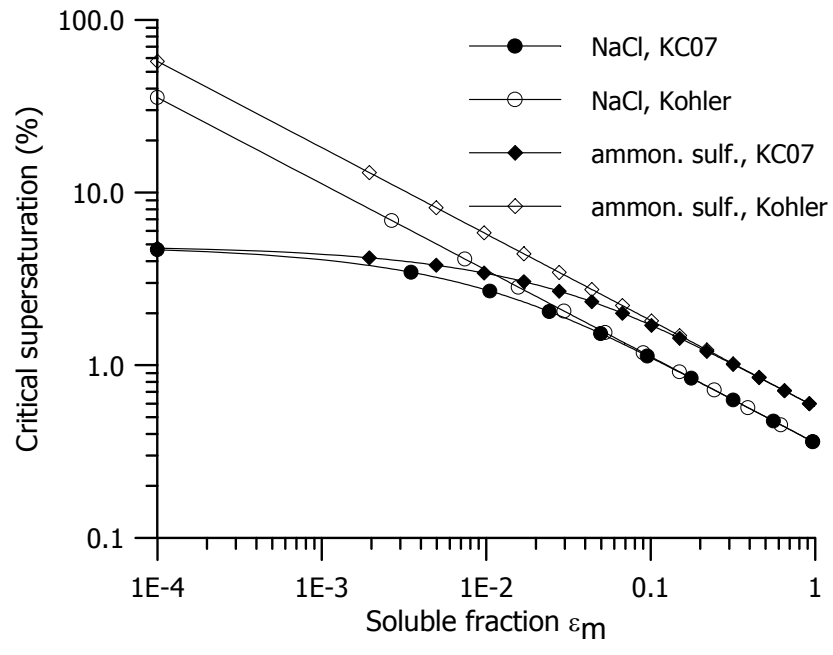

Fig. 2. Critical supersaturations calculated as a function of the soluble fraction $\varepsilon_{m}$ with analytical equations from $\mathrm{KC} 07$ (solid symbols) and in the approximation with the old Köhler's Eq. (17) here or (24) in KC07. The parameters are: particle radius $r_{d}=0.025 \mu \mathrm{m}$, soluble fraction is $\mathrm{NaCl}$ or ammonium sulfate as indicated in the legend.

gles), and compared with calculations here, both lines show a good correlation. However, this "dashed line" in Kok08 has another meaning than was interpreted in Kok08: a) it is the error in the critical radius but not in critical supersaturation as was stated in Kok08; b) it is the error not of $\mathrm{KCO} 7$ equation, but the error of the Köhler approximation relative to more general equation for $r_{c r}$ from $\mathrm{KC} 07$, and this error was already calculated in $\mathrm{KC} 07$. Therefore, a "comparison with KC07" with the dashed line in Kok08 was misleading, and Kok08's analysis could not draw any valid conclusions on the accuracy of the new $\mathrm{KC} 07$ equations comparing with the old Köhler equation.

The "solid line" from Fig. 1 in Kok08 is compared in Fig. 1d with the calculated error of the "low- $\varepsilon_{m}$ approximation" (Eq. 18 here or (30) in KC07). At soluble fraction $\varepsilon_{m} \leq 0.1$, both curves are sufficiently close, but the curves diverge at $\varepsilon_{m}>0.1$ : Kok08 goes steeply upward, and the curve of correct error goes down to negative values. Thus, the solid line from Kok08 described something different but not the error of the "low- $\varepsilon_{m}$ approximation"; hence it was also irrelevant for evaluation of $\mathrm{KC} 07$ and could not be a basis for any conclusions on its validity.

Figure 2 shows the critical supersaturations calculated with Eqs. (8)-(10) for $\mathrm{NaCl}$ and ammonium sulfate. They increase with decreasing soluble fraction, however, $\mathrm{KC} 07$ equations predict much slower increase than the Köhler's equation, which has good accuracy at $\varepsilon_{m}>0.05-0.1$ for $s_{c r}$ but fails at smaller $\varepsilon_{m}$. The $s_{c r}$ predicted by the KC07 solutions tend to quite reasonable values $\sim 4 \%$ even at very small $\varepsilon_{m}$, while $s_{c r}$ obtained from the classical Kohler solution reaches $35-60 \%$ at $\varepsilon_{m} \rightarrow 10^{-4}$. This indicates that aerosols 

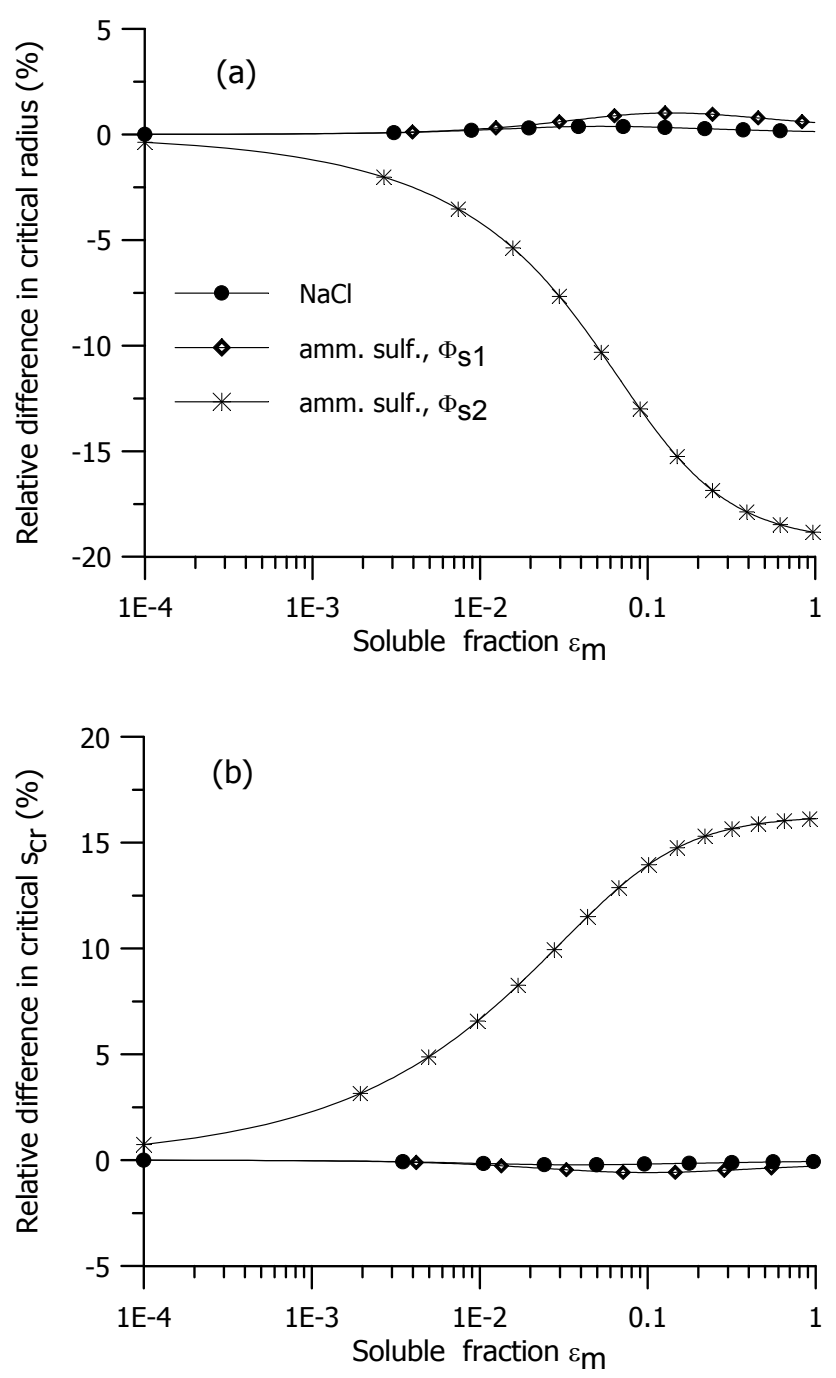

Fig. 3. The difference (\%) in critical radii (a) and supersaturations (b) as a function of the soluble fraction $\varepsilon_{m}$ calculated with analytical equations from $\mathrm{KC} 07$ and $\mathrm{Kok} 08$ as $\left(\mathrm{X}_{\mathrm{KC} 07-}\right.$ $\left.\mathrm{X}_{\mathrm{Kok} 08}\right) / \mathrm{X}_{\mathrm{KC} 07} \times 100 \%$. Here $\mathrm{X}$ denotes $r_{c r}$ or $s_{c r}$, and the subscript denotes the source work. Parameters are the same as in Fig. 1 and in Kokkola et al. (2008). The solid circles relate to $\mathrm{NaCl}$. The asterisks relate to ammonium sulfate calculated with dilute and ideal solution approximation as in Kok08, with $\Phi_{s 2}=1$, then the difference reaches $16-18 \%$. The diamonds relate to ammonium sulfate calculated with equations from Kok08 but corrected according to $\mathrm{KC} 07$, i.e., without assumption on dilute solution and $\Phi_{s 1}<1$ as in $\mathrm{KC} 07$, then the difference between both methods does not exceed $0.4-1 \%$.

with very small soluble fractions (e.g., mineral dust covered by a thin soluble film as observed by Levin et al., 1996) can serve as effective $\mathrm{CCN}$ at supersaturations that could be reached in convective updrafts. The dependencies of $r_{c r}$ and $s_{c r}$ on the initial radius $r_{d}$ and other parameters are described in $\mathrm{KC} 07$.
A correct comparison of calculations of $\mathrm{KC} 07$ with Kok08 is shown in Fig. 3. The values of $r_{c r}$ and $s_{c r}$ from $\mathrm{KC} 07$ were calculated using Eqs. (8)-(10). The $r_{c r}$ and $s_{c r}$ determined from Kok08 solutions were calculated in two ways: a) using the dilute and ideal solution approximation for $A_{F}, B_{F}$ in (5) and $\Phi_{s}=1$ as adopted in Kok08; b) with correction of Kok08 based on $\mathrm{KC} 07$, i.e., generalizing $A_{F}, B_{F}$ without the dilute approximation and with $\Phi_{s} \neq 1$ (for ammonium sulfate only), corresponding to $A_{K}, B$ in (2) as in $\mathrm{KC} 07$ solutions. The relative difference between the KC07 and Kok08 solutions were calculated as $\left(X_{\mathrm{KC} 07}-X_{\mathrm{Kok} 08}\right) / X_{\mathrm{KC} 07} \times 100 \%$, where $X$ denotes $r_{c r}$ or $s_{c r}$.

Figure $3 \mathrm{a}, \mathrm{b}$ shows that with Kok08 equations as they are, i.e., with dilute and ideal solution approximation in parameters in Kok08 for ammonium sulfate, the difference between KC07 and Kok08 does not exceed 16-18\% for both $r_{c r}$ and $s_{c r}$ (asterisks in Fig. 3a, b). Thus, a correct comparison shows that equations from Kok08 yield results close to $\mathrm{KC} 07$, and the 16-18\% discrepancy is associated with the use in Kok08 of the approximation of dilute and ideal solution. Note the error associated with the ideal solution approximation can be much greater for the substances with lower osmotic potentials or activity coefficients. If Kok08 equations are corrected following $\mathrm{KC} 07$, i.e., without approximations of dilute and ideal solution, the difference does not exceed $0.4-1 \%$ for both $r_{c r}$ and $s_{c r}$ and both $\mathrm{NaCl}$ and ammonium sulfate (circles and diamonds).

\section{Conclusions}

The analytical solutions for the critical radii $r_{c r}$ and supersaturations $s_{c r}$ for $\mathrm{CCN}$ activation derived in Khvorostyanov and Curry (2007) for the arbitrary CCN soluble fraction $\varepsilon_{m}$ are correct. This was proven here by direct substitution of the solution into the original equation and analysis of the original cubic equation for $r_{c r}$. These solutions convert into the classical Köhler's equations for the sufficiently high soluble fraction $\geq 0.1-0.2$, generalize them for arbitrary soluble fraction, and tend to the other new (non-Köhler's) limit for very small $\varepsilon_{m}$. This was proven by deriving the analytical limits from the general solution.

A comparison of analytical solutions for the critical radii and supersaturations of $\mathrm{CCN}$ with insoluble fractions obtained by Khvorostyanov and Curry (2007) and Kokkola et al. (2008) revealed the following. The analytical solution obtained in Kok08 for the critical diameter $D_{c r}$ has a form very similar to that in $\mathrm{KC} 07$, can be rewritten in a form that exactly repeats $\mathrm{KC} 07$, and represent a particular case of solutions from $\mathrm{KC} 07$ but with the additional approximations of an ideal and dilute solution adopted in Kok08.

A comparison of the corresponding numerical solutions revealed that the difference between $\mathrm{KC} 07$ and Kok08 is much smaller than $100 \%$ found in Kok08, and is caused mostly by the approximations made in Kok08. If equations 
from Kok08 are used as they are, i.e., in approximation of an ideal and dilute solution, then the difference between $\mathrm{KC} 07$ and Kok08 does not exceed 16-18\%. If equations from Kok08 are corrected following KC07, i.e., without assumption of solution ideality and high dilution, the difference does not exceed $0.4-1 \%$.

A conclusion made in Kok08 - that equations from $\mathrm{KC} 07$ are valid only for the low soluble fraction and that the difference between KC07 and Kok08 may exceed 100\% - was incorrect and based on erroneous calculations of the difference between the two works in Kok08. For some unclear reason, Kok08 compared their solutions not with the general solution from KC07, but with the Köhler's solution (dashed line in Fig. 1 in Kok08) or some unknown quantity (solid line in Fig. 1 in Kok08), which does not match any of the quantities from $\mathrm{KC} 07$, not even the "low soluble fraction" limit.

An analysis of the possible reasons of this difference between $\mathrm{KC} 07$ and Kok08 was additionally complicated by the misprint in the final equation for supersaturation in Kok08: the Eq. (8) for $s_{c r}$ in Kok08 was written with an erroneous sign "plus" instead of correct "minus" between the Kelvin and Raoult terms. The analytical and numerical study performed in this work, along with the interactive discussion at ACPD web site, allowed to establish the validity of the $\mathrm{KC} 07$ work, clarify the misprints and errors in Kok08, and to find the relation between the two works. Thus, the solutions for $r_{c r}$ and $s_{c r}$ obtained in $\mathrm{KC} 07$ are valid over a wide range of soluble fractions, and can be used for development of parameterization of drop activation on mixed $\mathrm{CCN}$, including the cases with very small soluble fractions. In particular, the two versions of such parameterizations were developed in $\mathrm{KC} 07$ and Khvorostyanov and Curry (2008).

Acknowledgements. This research has been supported by the NASA Modeling and Parameterization Program and NASA grant NNX07AQ26G. The authors are grateful to the two anonymous reviewers for reading the manuscript and useful remarks that allowed to improve the paper.

Edited by: A. Nenes

\section{References}

Charlson, R. J., Seinfeld, J. H., Nenes, A., Kulmala, M., Laaksonen, A., and Facchini, M. C.: Reshaping the theory of cloud formation, Science, 292, 2025-2026, 2001.

Foster, P., Ramaswamy, V., Artaxo, P., Berntsen, T., Betts, R., Fahey, D. W., Haywood, J., Lean, J., Lowe, D. C., Myhre, G., Nganga, J., Prinn, R., Raga, G., Schulz, M., and Van Dortland, R.: Changes in Atmospheric Constituents and in Radiative Forcing. Contribution of WG-I to the Fourth Assessment Report of the Intergovernmental Panel on Climate Change, Chapter 2, The Physical Science Basis, Cambridge University Press, 129-234, 2007.
Ghan, S., Chuang, C., and Penner, J.: A parameterization of cloud droplet nucleation. Part 1, Single aerosol species, Atmos. Res., 30, 197-222, 1993.

Ghan, S., Chuang, C., Easter, R., and Penner, J.: A parameterization of cloud droplet nucleation. Part 2, Multiple aerosol types, Atmos. Res., 36, 39-54, 1995.

Hänel, G.: The properties of atmospheric aerosol particles as functions of the relative humidity at thermodynamic equilibrium with the surrounding moist air, Adv. Geophys., 19, 73-188, 1976.

Khvorostyanov and Curry: Aerosol size spectra and CCN activity spectra: Reconciling the lognormal, algebraic and power laws, J. Geophys. Res., 111 D12202, doi:10.1029/2005JD006532, 2006.

Khvorostyanov, V. I. and Curry, J. A.: Refinements to the Köhler's theory of aerosol equilibrium radii, size spectra, and droplet activation: Effects of humidity and insoluble fraction. J. Geophys. Res., 112(D5), D05206, doi:10.1029/2006JD007672, 2007.

Khvorostyanov, V. I. and Curry, J. A.: Kinetics of cloud drop formation and its parameterization for cloud and climate models, J. Atmos. Sci., 65, 2784-2802, 2008.

Köhler, H.: The nucleus in the growth of hygroscopic droplets, Trans. Farad. Soc., 32, 1152-1161, 1936.

Kokkola, H.: Interactive comment on "Comment on Kokkola et al. (2008) - Comparisons with analytical solutions..., by V. I. Khvorostyanov and J. A. Curry", Atmos. Chem. Phys. Discuss., C1572-1574, 2009.

Kokkola, H., Vesterinen, M., Anttila, T., Laaksonen, A., and Lehtinen, K. E. J.: Technical note: Analytical formulae for the critical supersaturations and droplet diameters of CCN containing insoluble material. Atmos. Chem. Phys., 8, 1985-1988, 2008, http://www.atmos-chem-phys.net/8/1985/2008/.

Kulmala, M., Laaksonen, A., Korhonen, P., Vesala, T., and Ahonen, T.: The effect of atmospheric nitric acid vapor on cloud condensation nucleus activation, J. Geophys. Res., 98, 22949-22958, 1993.

Laaksonen, A., Korhonen, P., Kulmala, M., and Charlson, R. J.: Modification of the Köhler equation to include soluble trace gases and slightly soluble substances, J. Atmos. Sci., 55, 853862, 1998.

Levin, Z., Ganor, E., and Gladstein, V.: The Effects of Desert Particles Coated with Sulfate on Rain Formation in the Eastern Mediterranean, J. Appl. Meteorol., 35, 1511-1523, 1996.

Lohmann, U. and J. Feichter: Global indirect aerosol effects: A review, Atmos. Chem. Phys., 5, 715-737, 2005.

McFiggans, G., Artaxo, P., Baltensperger, U., Coe, H., Facchini, M. C., Feingold, G., Fuzzi, S., Gysel, M., Laaksonen, A., Lohmann, U., Mentel, T. F., Murphy, D. M., O’Dowd, C. D., Snider, J. L., and Weingartner, E.: The effect of physical and chemical aerosol properties on warm cloud droplet activation. Atmos. Chem. Phys., 6, 2593-2649, 2006.

Pruppacher, H. R. and J. D. Klett: Microphysics of Clouds and Precipitation, 2nd ed., Kluwer Academic Publishers: Boston, MA, USA, 954 pp., 1997.

Shulman, M. L., Jacobson, M. L., Charlson, R. J., Synovec, R. E., and Young, T. E., Dissolution behavior and surface tension effects of organic compounds in nucleating cloud droplets, Geophys. Res. Lett., 23, 277-280, 1996.

Seinfeld, J. H. and Pandis, S. N.: Atmospheric Chemistry and Physics, Wiley, New York, USA, 1326 pp., 1998. 